\title{
Cosmic acceleration without quintessence
}

\author{
Narayan Banerjee* and Diego Pavón ${ }^{\dagger}$ \\ Departamento de Física. Facultad de Ciencias. Edificio Cc. Universidad Autónoma de Barcelona. E-08193 Bellaterra
}

(Barcelona). Spain

()

\begin{abstract}
It is argued that the Brans-Dicke theory may explain the present accelerated expansion of the universe without resorting to a cosmological constant or quintessence matter.
\end{abstract}

\section{INTRODUCTION}

For several decades the general belief in cosmology had been in favour of a presently matter-dominated universe, expanding as $a \propto t^{2 / 3}$, where $a$ is the scale factor of the Robertson-Walker metric and $t$ is the cosmic time. The corresponding decelerated expansion $\left(q_{0}=-a \ddot{a} / \dot{a}^{2}=1 / 2\right)$ was more or less compatible with all the cosmological tests. The problems with the standard cosmology were confined to the early stages of the evolution of the universe and they were expected to be taken care of by the inflationary paradigm. But the recent observations regarding the luminosity-redshift relation of the type Ia supernovae up to about $z=1$ revealed that the universe is in fact expanding at a faster rate, even possibly with an acceleration $\left(q_{0}<0\right)$ [1]. This observation naturally leads to the search for the matter fields, hitherto unknown or neglected, which could introduce such a non-decelerated expansion. This matter is called a quintessence matter ( Q-matter for short). The list of possible candidates, being explored as Q-matter, consists of a cosmological constant or a time dependent cosmological term, a scalar field with a potential giving rise to a negative pressure [2] at the present epoch, a dissipative fluid yielding an effective negative stress [3] or more exotic matter like a frustrated network of non-Abelian cosmic strings or a frustrated network of domain walls 鿉. Amongst the scalar fields chosen as the Q-matter, the "tracker" field rolling down its potential [5] appears to be the most attractive. But most of these investigations are effective only for a spatially flat $(k=0)$ model and thus the fine tuning or the flatness problem remains an unsolved one. The exception is the recent work by Chimento et al. [3], where a combination of a quintessence scalar field with a potential and a fluid with a bulk viscous stress has been shown to work for $k=-1$. This approach also solves the coincidence problem in the sense that the ratio of the density parameters for the normal matter and the scalar field asymptotically becomes a constant.

The aim of this paper is to show that Brans-Dicke's scalar tensor theory can potentially solve the quintessence problem as it can lead to non-decelerating solutions for the scale factor for the present universe dominated by cold matter with negligible pressure. We would like to stress that this kind of solutions can be obtained without invoking a Q-matter or even dissipative processes. For a spatially flat model $(k=0)$ one can indeed have an accelerated expansion, and even for a non-flat $(k \neq 0)$ model, one can at least have a non-decelerated expansion. Very recently Bertolami and Martins [6] presented an accelerating model for the spatially flat $(k=0)$ in a modified Brans-Dicke theory using a potential which is a function of the Brans-Dicke scalar field itself. In this work we show that this solution also can be obtained in Brans-Dicke theory without the potential. However, there are problems in bridging this result with the radiation-dominated decelerated universe for the same values of the Brans-Dicke parameter $\omega$. In the next section we show that the solution to this problem lies in the natural generalization of the theory by allowing $\omega$ to be a function of the Brans-Dicke scalar field.

\section{FIELD EQUATIONS AND SOLUTIONS}

For a Friedmann-Robertson-Walker spacetime, with scale factor $a(t)$, spatial curvature index $k$, and assuming that the only matter-field is a perfect fluid, the gravitational field equations in Brans-Dicke theory are

\footnotetext{
*Permanent address: Relativity and Cosmology Research Centre, Department of Physics, Jadavpur University, Calcutta700032 , India

${ }^{\dagger}$ Electronic mail address: diego@ulises.uab.es
} 


$$
\begin{gathered}
3 \frac{\dot{a}^{2}+k}{a^{2}}=\frac{\rho_{f}}{\phi}-3 \frac{\dot{a} \dot{\phi}}{a \phi}+\frac{\omega}{2} \frac{\dot{\phi}^{2}}{\phi^{2}}, \\
2 \frac{\ddot{a}}{a}+\frac{\dot{a}^{2}+k}{a^{2}}=\frac{-p_{f}}{\phi}-\frac{\omega}{2} \frac{\dot{\phi}^{2}}{\phi^{2}}-2 \frac{\dot{a} \dot{\phi}}{a \phi}-\frac{\ddot{\phi}}{\phi},
\end{gathered}
$$

where $\omega$ is the Brans-Dicke's constant parameter, while $\rho_{f}$ and $p_{f}$ are the density and hydrostatic pressure respectively of the fluid distribution. These two latter quantities are connected by a barotropic equation of state $p_{f}=\left(\gamma_{f}-1\right) \rho_{f}$, $\gamma_{f}$ being the (constant) adiabatic index of the fluid.

The wave equation for the Brans-Dicke scalar field reads

$$
\ddot{\phi}+\frac{3 \dot{a} \dot{\phi}}{a}=\frac{\rho_{f}-3 p_{f}}{2 \omega+3},
$$

and this combined with the field equations ( 1) and (2) leads to the matter conservation equation

$$
\dot{\rho}_{f}+3 \frac{\dot{a}}{a}\left(\rho_{f}+p_{f}\right)=0 .
$$

Assuming that at the present epoch the universe is filled with cold matter of negligible pressure (i.e., dust), we put $p_{f}=0$ in the last equation and obtain the relation

$$
\rho_{f}=\rho_{0} a^{-3}
$$

where $\rho_{0}$ is a constant of integration. With $p_{f}=0$ and $\rho_{f}$ being given by equation ( 5 ), the wave equation becomes

$$
\left(a^{3} \dot{\phi}\right)^{\cdot}=\frac{\rho_{0}}{2 \omega+3},
$$

which immediately yields the first integral

$$
a^{3} \dot{\phi}=\frac{\rho_{0} t}{2 \omega+3} .
$$

We are primarily interested in a power law non-decelerating solution for the scale factor in keeping with the recent observations. So we take

$$
a=a_{0} t^{\alpha} \quad(\alpha \geq 1)
$$

where $a_{0}$ is a positive-definite constant.

Equation (6) can now be integrated for $\phi$ as

$$
\phi=\frac{\rho_{0} t^{2-3 \alpha}}{a_{0}^{3}(2 \omega+3)(2-3 \alpha)} .
$$

Inspection of the field equations ( 1) and (2) reveals that when $k \neq 0$, the only possible solution in this simple power function form is that with $\alpha=1$, i.e.,

$$
a=a_{0} t
$$

and

$$
\phi=-\frac{\rho_{0}}{a_{0}^{3}(2 \omega+3) t} .
$$

So one has a scenario where the present deceleration parameter vanishes $\left(q_{0}=0\right)$, i.e., today the Universe is in a state of uniform expansion. Although this very simple model does not yield an accelerating universe for a non-zero $k$, it expands with no deceleration either and may sufficiently fulfil the requirements set by the recent observations on the distant supernovae [7].

From (2) the consistency condition (for $\alpha=1$, i.e., $q_{0}=0$ ) 


$$
\omega=-2\left(1+\frac{k}{a_{0}^{2}}\right)
$$

follows. The latter equation indicates that at least for $k=0$ or $k=+1, \omega$ is negative. This is perfectly consistent with equation ( 10) which requires that $\omega<-3 / 2$ as $\phi$ is a positive quantity.

If the universe is spatially flat $(k=0)$, this model yields several possibilities including accelerated expansions for the universe. With $k=0$, the equation ( 6 ), put back in the field equations yields for dust $\left(p_{f}=0\right)$ the general solution as $[8=2(\omega+1) /(3 \omega+4)$, i.e.,

$$
a=a_{0} t^{2(\omega+1) /(3 \omega+4)}
$$

and the scalar field takes the form

$$
\phi=\frac{(3 \omega+4) \rho_{0} t^{2 /(3 \omega+4)}}{2 a_{0}^{3}(2 \omega+3)} .
$$

Now it is easily seen that for different negative values of $\omega$, one can generate different accelerating solutions. Such as, for $\omega=-5 / 3$, we get $\alpha=4 / 3$ and $\phi \propto t^{-2}$, which is the solution presented by Bertolami and Martins [6]. In this case, $q_{0}=-1 / 4$. For $\omega=-8 / 5, \alpha$ becomes $3 / 2$ and $\phi \propto t^{-5 / 2}$, giving $q_{0}=-1 / 3$, i.e., an accelaration rate higher than the Bertolami-Martins' solution. For different values of $\omega$ in the range $-2 \leq \omega \leq-3 / 2$, this model yields a host of accelerating solutions for a spatially flat universe. In fact this negative value of $\omega$ explains the behaviour of the model. A sufficiently negative $\omega$ may effectively lead to a negative pressure (see equation (2)) and thus drive a positive acceleration or uniform expansion without the violation of the energy condition by normal matter.

The solutions and the corresponding value of $\omega$ can be used in equation ( 1) to calculate the age $t_{0}$ of the universe. It turns out that the age is of the order of $H_{0}^{-1}$. For the uniformly expanding spatially flat universe, $t_{0}$ is exactly equal to $H_{0}^{-1} \sim 1.62 \times 10^{10} \mathrm{yr}$. The higher the acceleration, the older the universe is. For $q_{0}=-1 / 4$, $t_{0}=\frac{4}{3} H_{0}^{-1}$.

As Brans-Dicke theory is a varying $G$ theory, the rate of this variation has been checked in this model and it appears to be compatible with the observational limit. For instance, with $q_{0}=-1 / 4,(\dot{G} / G)_{0}=3 H_{0} / 2<2.5 \times 10^{-10}$ which is safely below the upper limit of $4 \times 10^{-10} \mathrm{yr}^{-1}$ set by observations (see [9] and references therein).

It is important to note that although for the some negative values of $\omega$, the model can lead to decelerating expansions for the Universe when it is radiation-dominated, in that case the value of $\omega$ would be less negative than $-3 / 2$, i.e., $-3 / 2 \leq \omega \leq 0$. The indicated range of values of $\omega$, i.e., $-2 \leq \omega \leq-3 / 2$, which drives an accelerated expansion for a matter-dominated model, does not produce a consistent model with the radiation-dominated epoch. Thus the suggested model seems to badly spoil the big-bang nucleosynthesis scenario. One way out of this problem is to start with a modified version of Brans-Dicke theory where the parameter $\omega$ is a function of the scalar field $\phi$ rather than a constant [10]. In this case equations (11), (2) and (4) remain in place, but the wave equation for the scalar field (3) has an additional term and becomes

$$
\ddot{\phi}+\frac{3 \dot{a} \dot{\phi}}{a}=\frac{\rho_{f}-3 p_{f}}{2 \omega+3}-\frac{\dot{\omega} \dot{\phi}}{2 \omega+3} .
$$

With the equation of state for radiation $\left(p_{f}=\frac{1}{3} \rho_{f}\right)$, equation (14) immediately gives a first integral

$$
\dot{\phi}(2 \omega+3)^{1 / 2}=\frac{A}{a^{3}}
$$

where $A$ is an integration constant. It turns out that there are functional forms of $\omega$ for which the universe expands with a deceleration such as like $a \propto t^{\frac{1}{2}}$ in this case so that the primordial nucleosynthesis can be successfully explained. A simple choice like

$$
2 \omega+3=(\phi-1)^{2}
$$

when used in equation (15) along with $a \propto t^{1 / 2}$ yields the integral

$$
(\phi-1)^{2}=\frac{B}{\sqrt{t}},
$$


$B$ being a constant of integration. Equations (16) and (17) clearly shows that $\omega$ decreases with time towards a constant value $-3 / 2$, which indeed produces an accelerated expansion for a late dust-dominated universe as we have seen.

In general, a choice of $\omega$ as a polynomial in $\phi$ is

$$
2 \omega+3=\sum_{i=1}^{n} A_{i} \phi^{n_{i}},
$$

where $n_{i} \geq 0$ and $A_{i}$ 's are constants appears to solve this problem. A varying $\omega$ theory with a nonminimal coupling between gravity and the scalar field different from the Brans-Dicke theory $\left(\phi^{2} R\right.$ as opposed to $\phi R$ in BDT) may also explain the late time behaviour of the universe as pointed out very recently by Bartolo and Pietroni [11. It is also worthwhile to note that Sen and Seshadri [12] have shown that with small negative values of $\omega$, it is possible to obtain growing modes for the density perturbations for a universe with a power law expansion rate.

\section{A CONFORMALLY TRANSFORMED VERSION}

In Brans-Dicke theory, the folklore is that $\omega$ is positive, or at least larger than $-3 / 2$. There are two reasons for this belief. The first reason is that in the weak field limit, the Newtonian constant of gravitation $\mathrm{G}$ is given by,

$$
G=\left(\frac{2 \omega+3}{2 \omega+4}\right) \frac{1}{\phi},
$$

and for $-2<\omega<-3 / 2, \mathrm{G}$ becomes negative. But it should be emphasized that this is only in a weak field limit. So in the model presented in the present work, $\mathrm{G}$ does not become negative, only this weak field approximation does not hold. In the full non linear Brans-Dicke theory, the effective Gravitational constant is actually given by

$$
G=\frac{G_{0}}{\phi},
$$

which is indeed positive as $\phi$ is positive. It deserves mention that for the accelerating solution obtained by Bertolami and Martins [6] also, $\omega$ is actually negative.

The second reason for the belief that $\omega>-3 / 2$ is that the energy related to this scalar field is proportional to $(2 \omega+3)$. To see this, it is better to effect a conformal transformation

$$
\bar{g}_{\mu \nu}=\phi g_{\mu \nu}
$$

In the transformed version, $\mathrm{G}$ becomes a constant, but one has to sacrifice the equivalence principle as the rest mass of a test particle becomes a function of the scalar field [13]. So the geodesic equations are no longer valid and naturally the physical significance of different quantites are indeed questionable in this version of the theory. Nevertheless the equations in this version gives an insight in comparing the energies of different components of matter. The equations (11)-(2) in the new frame look like

$$
\begin{gathered}
\frac{3\left(\dot{\bar{a}}^{2}+k\right)}{\bar{a}^{2}}=\bar{\rho}_{f}+\frac{2 \omega+3}{4} \dot{\psi}^{2}, \\
\frac{2 \ddot{\bar{a}}}{\bar{a}}+\frac{\dot{\bar{a}}^{2}+k}{\bar{a}^{2}}=-\bar{p}_{f}-\frac{2 \omega+3}{4} \dot{\psi}^{2},
\end{gathered}
$$

and the wave equation ( 3) transforms to

$$
\ddot{\psi}+3 \frac{\dot{\bar{a}} \dot{\psi}}{\bar{a}}=\frac{\bar{\rho}_{f}-3 \bar{p}_{f}}{2 \omega+3}
$$

where an overhead bar indicates quantities in the new frame, $\psi=\ln \phi$, and $\bar{a}^{2}=\phi a^{2}$. The density and the pressure of the normal matter in this version are related to those in the original version in a simple way as $\bar{\rho}_{f}=\phi^{-2} \rho_{f}$ and $\bar{p}_{f}=\phi^{-2} p_{f}$. It is clearly seen that the contribution to the energy density by the scalar field is given by 


$$
\bar{\rho}_{\phi}=\frac{2 \omega+3}{4} \dot{\psi}^{2}
$$

Here $\psi$ looks like a massless minimally coupled scalar field and at least formally behaves as a perfect fluid with a stiff equation state, $\bar{p}_{\phi}=\bar{\rho}_{\phi}$. (Note that the corresponding adiabatic index is $\gamma_{\phi}=2$ ). Equation ( 22) explains why $\omega$ is usually taken to be larger than the $-3 / 2$ from the consideration of the positivity of energy.

Our intention is to get a sufficiently negative pressure from some source so that we can get $q_{0} \leq 0$, and here we achieve that by means of a scalar field $\phi$ which has a negative energy. But in our case $\phi$ is a geometric field unlike the non-gravitational fields such as normal matter or the quintessence field, and so its having a negative energy is not pathological in that sense. We rather save the normal matter from the unappealing feature of a negative pressure and yet get a non positive-definite deceleration parameter.

This conformally transformed version of the theory gives an insight regarding the solution of the flatness problem too. The density parameter is defined as $\bar{\Omega}=\bar{\rho} / 3 \bar{H}^{2}$, where

$$
\bar{\rho} \equiv \bar{\rho}_{f}+\bar{\rho}_{\phi}=\bar{\rho}_{f}+\frac{2 \omega+3}{4} \dot{\psi}^{2}
$$

is the total energy density. The subscripts $f$ and $\phi$ refer to the normal fluid and the scalar field, respectively. $\bar{H} \equiv \dot{\bar{a}} / \bar{a}$ is the Hubble parameter in the new frame.

The Bianchi identity yields the relation

$$
\dot{\bar{\rho}}+3 \gamma \bar{H} \bar{\rho}=0
$$

where $\gamma$ is the average barotropic index defined by the relation

$$
\gamma \bar{\Omega}=\gamma_{f} \bar{\Omega}_{f}+\gamma_{\phi} \bar{\Omega}_{\phi}
$$

where $\bar{\Omega} \equiv \bar{\Omega}_{f}+\bar{\Omega}_{\phi}$.

Using equations (19) and (23) one can write

$$
\dot{\bar{\Omega}}=\bar{\Omega}(\bar{\Omega}-1)(3 \gamma-2) \bar{H}
$$

This equation shows that $\bar{\Omega}=1$ is indeed a solution, but $(\partial \dot{\bar{\Omega}} / \partial \bar{\Omega})_{H}$ must be negative at $\bar{\Omega}=1$ if this $\bar{\Omega}$ value is to be a stable solution of (25). This requires that $\gamma$ should be less than $2 / 3$. As the ratio of the density to pressure of the fluid remains the same in the two versions of the theory, the adiabatic index $\gamma_{f}$ also remains the same. Because of our choice of $\bar{p}_{f}=0$ and $\bar{p}_{\phi}=\bar{\rho}_{\phi}$, we have $\gamma_{f}=1$ and $\gamma_{\phi}=2$. So from equation ( 24) one has

$$
\gamma=\frac{\bar{\Omega}_{f}+2 \bar{\Omega}_{\phi}}{\bar{\Omega}_{f}+\bar{\Omega}_{\phi}} .
$$

As $2 \omega+3$ is negative, $\bar{\Omega}_{\phi}$ is also negative and one can achieve $\gamma<2 / 3$ provided

$$
\bar{\Omega}_{f}<4\left|\bar{\Omega}_{\phi}\right|
$$

So $\bar{\Omega}=1$ is indeed a stable solution in this model depending on the relative magnitudes of the energies of matter and the Brans-Dicke's scalar field. With $\bar{\Omega}=1$, we have $\bar{\Omega}_{k}=-k / \bar{a}^{2}=0$ and thus the flatness problem can be solved.

It is true that although the conformally transformed version (popularly known as the Einstein frame) is appealing from the point of view of the computational simplicity, the quantities lose their physical significance for reasons stated at the beginning of this section. But it must be emphasized that the character of $k$ remains the same and if it is zero in this version, it is so in the physical original version of the theory as well. For a spatially flat universe $(k=0)$ we can thus construct accelerating models for the Universe and reproduce the Bertolami-Martins solution as a special case and we do not have to invoke an additional self-interaction term for this. Even for the non-flat cases, we can at least obtain a non-decelerating model with $q_{0}=0$, whereas almost all the quintessence models produce solutions only for a spatially flat universe with the only exception of the dissipative fluid model recently discussed by Chimento et al. [3], which works for open universes as well. For a discussion of the flatness problem in Brans-Dicke theory see the paper by Levine and Freese [14]. 


\section{CONCLUDING REMARKS}

Brans-Dicke theory proved to be extremely useful in solving some of the outstanding problems in the inflationary universe scenario with the possibility of an "extended inflation" [15]. And now although the theory might relinquish some of its natural attraction as it fails to produce general relativity as an infinite $\omega$ limit [16] contrary to the earlier belief, once again it appears to be able to account for some outstanding features of our present Universe like the accelerated expansion and the coincidence problem by its own right without having to invoke dissipative processes or exotic fields.

It should not be overlooked, however, that $\omega$ has to pick up a low negative value (i.e., $-\omega \sim \mathcal{O}(1)$ ) in order to solve the acceleration and coincidence problems by a single stroke (e.g., $q_{0}=-1 / 4$ implies $a \propto t^{4 / 3}$ and consequently $\omega=-5 / 3)$. This squarely conflicts with the lower limit imposed on $|\omega|$ by solar system experiments, namely $|\omega| \geq 500$ [17]. Nevertheless in "extended inflation" the model of La and Steinhardt worked provided that $\omega$ takes a value close to 20 [15] which is also embarrasingly lower than the mentioned astronomical limit. Therefore it remains a problem to find a suitable compromise between astronomical observations and cosmological requirements. Another problem is that the constant negative value of $\omega$ does not produce a consistent radiation model which explains the primordial nucleosynthesis. We have shown that a varying $\omega$ theory like Nordtverdt's [10] can give rise to a decelerating radiation model where the big-bang nucleosynthesis scenario is not adversedly affected and $\omega$ evolves to the small and negative constant values required for the late time acceleration in the matter-dominated epoch. A thorough survey of varying $\omega$ theories is perhaps warranted which may bridge the decelerating radiation universe with the accelerating matter universe while the local inhomogeneities might locally give rise to high values of $\omega$ to be consistent with astronomical experiments.

However, attempts to solve the aforesaid problems outside Brans-Dicke theory do not fare much better. The introduction of a cosmological constant of the order of the critical density lacks of a solid support from quantum field theory [18], and arguments based on the anthropic principle are anything but convincing [19]. On their part models based on quintessence suffer from the problem of unwanted long-range forces and that they cannot be as homogeneous as it should [20].

\section{ACKNOWLEDGMENTS}

The authors thank Winfried Zimdahl for a critical reading of an earlier version of this paper and valuable comments. This work has been partially supported by Spanish Ministry of Education under Grant PB94-0781. One of us (NB) is grateful to the "Direcció General de la Recerca" of the Catalonian Government for financial support under grant PIV99.

[1] S. Perlmutter et al., Astrophys. J. 517, 565 (1999); A.G.Riess et al., Astron. J. 116, 1009 (1998); P.M. Garnavich et al., Astrophys. J. 509, 74 (1998).

[2] R.R. Caldwell, R. Dave and P.J. Steinhardt, Phys. Rev. Lett. 80, 1582 (1998); M. S. Turner and M. White, Phys. Rev. D 56, R4439 (1997).

[3] L.P. Chimento, A.S. Jakubi and D. Pavón, Phys Rev. D ( in press) astro-ph/0005070.

[4] M. Bucher and D. Spergel, Phys. Rev. D 60, 043505 (1999); M. Bucher, astro-ph/9908047.

[5] I. Zlatev, L. Wang and P.J. Steinhardt, Phys. Rev. Lett. 82, 896 (1999); P.J. Steinhardt, L. Wang and I. Zlatev, Phys. Rev. D 59, 123504 (1999).

[6] O. Bertolami and P.J. Martins, Phys. Rev. D 61, 064007 (2000).

[7] A.G. Riess, "The case for an accelerating universe from supernovae", astro-ph/0005229.

[8] C.H. Brans and R.H. Dicke, Phys. Rev. 124, 925 (1961).

[9] S. Weinberg Gravitation and Cosmology (J. Wiley, N.Y., 1972).

[10] K. Nordtvedt Jr., Astrophys. J. 161, 1059 (1970).

[11] N. Bartolo and M. Pietroni, Phys. Rev. D, 61, 023518 (1999).

[12] S. Sen and T.R. Seshadri, "Self interacting Brans Dicke cosmology and quintessence", gr-qc/0007079].

[13] R.H. Dicke, Phys. Rev. 125, 2163 (1962).

[14] J.J. Levin and K. Freese, Nucl. Phys. B 421, 635 (1994). 
[15] C. Mathiazhagan and V.B. Johri, Class. Quantum Grav. 1, L29 (1984); D. La and P.J. Steinhardt, Phys. Rev. Lett 62, 376 (1989).

[16] N. Banerjee and S. Sen, Phys. Rev. D, 56, 1334 (1997); V. Faraoni, ibid. 59, 084021 (1999).

[17] C.M. Will, Theory and Experiment in Gravitational Physics, (Cambridge University Press, 3rd edition, Cambridge, 1993).

[18] S. Weinberg, Rev. Mod. Phys. 61, 1 (1989); S.M. Carroll, "The Cosmological Constant" (submitted) astro-ph/0004075].

[19] S. Weinberg, "The Cosmological Constant Problem", Talk given at Dark Matter 2000, astro-ph/0005265.

[20] S.M. Carroll, Phys. Rev. Lett. 81, 3067 (1998). 